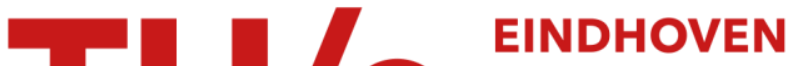 \\ UNIVERSITY OF \\ TECHNOLOGY
}

\section{Fiber-based broadband wireless access employing optical frequency multiplication}

Citation for published version (APA):

Garcia Larrode, M., Vegas Olmos, J. J., \& Koonen, A. M. J. (2006). Fiber-based broadband wireless access employing optical frequency multiplication. IEEE Journal of Selected Topics in Quantum Electronics, 12(4), 875881. https://doi.org/10.1109/JSTQE.2006.876173

DOI:

10.1109/JSTQE.2006.876173

Document status and date:

Published: 01/01/2006

\section{Document Version:}

Publisher's PDF, also known as Version of Record (includes final page, issue and volume numbers)

\section{Please check the document version of this publication:}

- A submitted manuscript is the version of the article upon submission and before peer-review. There can be important differences between the submitted version and the official published version of record. People interested in the research are advised to contact the author for the final version of the publication, or visit the $\mathrm{DOI}$ to the publisher's website.

- The final author version and the galley proof are versions of the publication after peer review.

- The final published version features the final layout of the paper including the volume, issue and page numbers.

Link to publication

\section{General rights}

Copyright and moral rights for the publications made accessible in the public portal are retained by the authors and/or other copyright owners and it is a condition of accessing publications that users recognise and abide by the legal requirements associated with these rights.

- Users may download and print one copy of any publication from the public portal for the purpose of private study or research.

- You may not further distribute the material or use it for any profit-making activity or commercial gain

- You may freely distribute the URL identifying the publication in the public portal.

If the publication is distributed under the terms of Article 25fa of the Dutch Copyright Act, indicated by the "Taverne" license above, please follow below link for the End User Agreement:

www.tue.nl/taverne

Take down policy

If you believe that this document breaches copyright please contact us at:

openaccess@tue.nl

providing details and we will investigate your claim. 


\title{
Fiber-Based Broadband Wireless Access Employing Optical Frequency Multiplication
}

\author{
M. García Larrodé, Student Member, IEEE, A. M. J. Koonen, Senior Member, IEEE, \\ and J. J. Vegas Olmos, Student Member, IEEE
}

\begin{abstract}
Employing optical frequency multiplication, we demonstrate experimentally the simultaneous delivery of different 64QAM radio signals at 5.8 and $17.7 \mathrm{GHz}$ after $4.4 \mathrm{~km}$ of multimode fiber in a single radio-over-fiber link. We also propose a flexible mechanism for the dynamic radio link adaptation, and the simultaneous transmission of an in-band control channel with the wireless data channels for power control purposes at the antenna site is demonstrated experimentally.
\end{abstract}

Index Terms-Broadband wireless access, optical fiber communications, radio-over-fiber systems.

\section{INTRODUCTION}

I $\mathrm{N}$ the last few years, broadband access has experienced an explosive boom with the demand for higher data rates to accommodate new services growing rapidly ever since. On the one side, we have witnessed a massive penetration of X-DSL and cable internet access in business and household environments. Fiber-to-the-home (FTTH) deployments are nowadays also becoming very popular as a future-proof infrastructure to provide high speed and triple play support, comprising both switched Ethernet-based and passive optical network (PON)-based architectures [1]. On the other side, wireless LAN systems like IEEE $802.11 \mathrm{a} / \mathrm{b} / \mathrm{g}$ have made broadband wireless access a reality, with the proliferation of cheap and easy-to-deploy access points in buildings, airports, shopping malls, and households. Emerging broadband fixed wireless access systems like IEEE 802.16 aim to enhance these broadband capabilities with innovative approaches in the sub-11 GHz band and in the millimeter wave region [2].

On the combination of both the high capacity of fiber optics and the wireless access flexibility, radio-over-fiber (RoF) distribution antenna systems provide a smooth merging of these two, traditionally opposite, approaches. The RoF distribution antenna systems have been identified as a flexible, bandwidth-efficient, and cost-effective option for fiber-based wireless access infrastructure, especially in in-building and business environments [3]. They enable the consolidation of the radio access control and signal processing at a centralized control station (CS) and the delivery of the radio signals transparently to the simplified antenna sites (AS) via optical fiber. The RoF link lies within the physical layer of the wireless system to be supported, and thus

Manuscript received August 14, 2005; revised March 24, 2006. This work was supported in part by the Dutch Ministry of Economics Affairs under the IOP GenCom Program.

The authors are with the COBRA Research Institute, Eindhoven University of Technology, 5600 MB Eindhoven, The Netherlands (e-mail: m.garcia. larrode@tue.nl; a.m.j.koonen@tue.nl; j.j.vegas@tue.nl).

Digital Object Identifier 10.1109/JSTQE.2006.876173 becomes an extension of the radio domain access. In this way, the radio characteristics and the wireless system requirements determine the RoF distribution antenna system design.

For the emerging broadband wireless systems, operating at carrier frequencies beyond $5 \mathrm{GHz}$, one of the main challenges of RoF techniques is the generation and delivery of highmicrowave signals at the AS, while maintaining the link simplicity. Different methods have been developed for this purpose [4]-[8]. These methods mainly focus on the RoF transmission system performance of microwave carriers and typical radio signal modulation formats. In addition to the transparent delivery of the radio signals to the AS, and, as a further step toward a reliable RoF infrastructure for broadband wireless access, other networking aspects have to be taken into account. First, different wireless standards will have to coexist at the same AS in a costeffective broadband wireless access infrastructure. Therefore, multistandard wireless access support by a single RoF link has to be guaranteed. Second, the RoF link has to be transparent to the radio channel variations and to the dynamic radio link adaptation strategies performed by the different wireless systems.

In this paper, we try to tackle these two aspects by employing the optical frequency multiplication (OFM) principle, proposed in [8]. OFM is a cost-effective method to optically generate microwave frequencies and deliver wireless signals to a remote AS, by the introduction of radio signals at relatively low frequencies from the CS [9]. It can be deployed with both single-mode (SMF) and multimode (MMF) fiber links, and it is transparent to different modulation formats [10].

This paper is organized as follows. Section II presents briefly the basic OFM principle and the maximum RF bandwidth capacity allowed by this technique. In Section III, the wireless multistandard support by a single OFM link is experimentally demonstrated with the simultaneous transmission of two different wireless signals recovered at different frequency bands. Section IV analyzes how an RoF link can affect the performance of a wireless link with dynamic radio link adaptation, and where this adaptation can take place. It also proposes and experimentally demonstrates the simultaneous transmission of a control channel with the wireless data channel over the same RoF link for power control purposes. Finally, Section V summarizes the main conclusions of this paper.

\section{RoF LINKS EMPLOYING OFM}

\section{A. OFM Principle}

The OFM principle is based on harmonics generation by frequency modulation (FM) to intensity modulation (IM) 


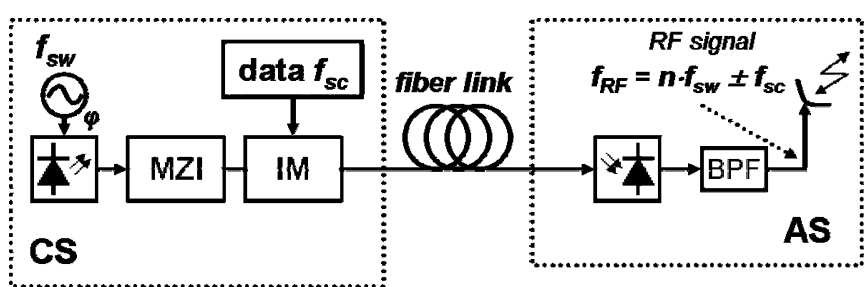

(a)

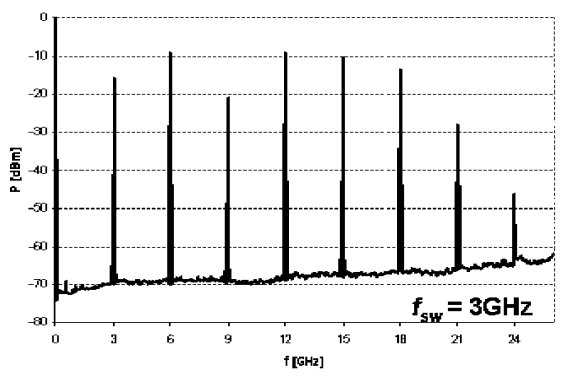

(b)

Fig. 1. OFM. (a) Basic scheme. (b) Generation of harmonics $\left(f_{\mathrm{sw}}=3 \mathrm{GHz}\right)$.

conversion, and is depicted in Fig. 1(a). At the CS, a continuous wave laser source is frequency modulated by a sinusoid with sweep frequency $f_{\mathrm{sw}}$; this optical FM signal is passed through a Mach-Zehnder interferometer (MZI), launched into the optical fiber link and recovered at the AS by a photodetector. At the output of the photodetector, radio frequency components at every harmonic of the sweep frequency $f_{\mathrm{sw}}$ are obtained [Fig. 1(b)], with relative amplitudes depending on $f_{\text {sw }}$, the FM index and the free spectral range (FSR) of the MZI. On top of the optical FM, a radio signal (data) put onto a low-frequency subcarrier $f_{\mathrm{sc}}$ can be employed to modulate the intensity of the swept light source at the CS by an intensity modulator. Then, this radio signal is recovered after the photodetector along with all the generated harmonics at $f_{\mathrm{RF}}=n f_{\mathrm{Sw}} \pm f_{\mathrm{Sc}}$ ( $n$ indicates the $n$th harmonic), at the AS. The desired $f_{\mathrm{RF}}$ signal can be selected with an adequate bandpass filter and conveyed to the antenna for the RF transmission.

In this way, high-frequency microwave carriers can be obtained by remotely generating radio signals at relatively low frequencies. Moreover, in comparison with the direct transmission of microwave carriers over a fiber link, which suffers carrier suppression at specific fiber lengths due to chromatic dispersion, OFM presents a higher dispersion tolerance, thanks to the FM to IM conversion performed with the MZI, which enhances the generation of higher order harmonics through a dispersive element [11]. This is especially interesting in multimode fiber (MMF) links, where the transmission bandwidth for direct modulated microwave carriers is dramatically limited by modal dispersion. With OFM, microwave carriers beyond the nominal bandwidth of MMF can be remotely generated.

\section{B. RF Bandwidth Capacity Characterization}

As explained above, in an OFM-RoF link, several harmonics of the sweep frequency $f_{\mathrm{sw}}$ are generated at the photodiode output, carrying, double-sided, the radio signal subcarriers $f_{\mathrm{sc}}$

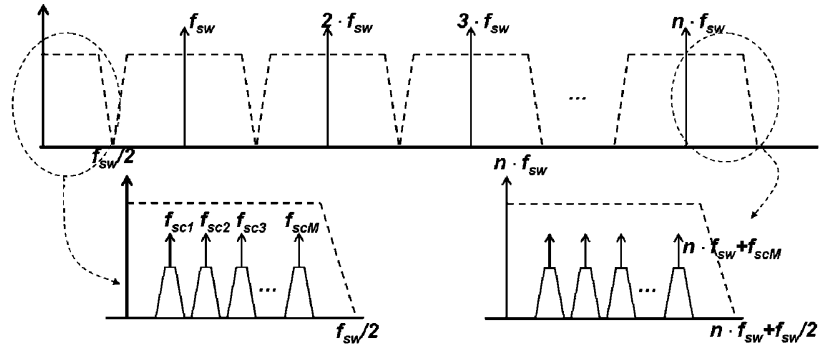

(a)

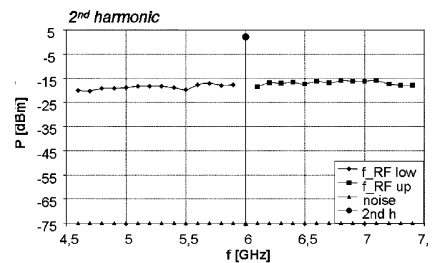

(b)

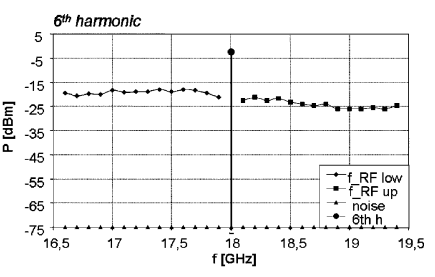

(c)
Fig. 2. RF bandwidth capacity employing OFM. (a) Harmonics carrying sided bands after photodetection. (b) Measurement of the second harmonic RF bandwidth $\left(f_{\mathrm{sw}}=3 \mathrm{GHz}\right.$, second harmonic $\left.=6 \mathrm{GHz}\right)$. (c) Measurement of the sixth harmonic RF bandwidth $\left(f_{\mathrm{sw}}=3 \mathrm{GHz}\right.$, sixth harmonic $\left.=18 \mathrm{GHz}\right)$.

along with each generated harmonic. Thus, the maximum RF bandwidth supported by one wavelength is limited by half the sweep frequency $f_{\mathrm{sw}}$. As depicted in Fig. 2(a), data channels on subcarriers below $f_{\mathrm{sw}} / 2$ can be used to modulate the intensity of the swept light source. After the photodetector, they can be separately recovered by filtering out the desired subcarrier. If those channels exceed $f_{\mathrm{sw}} / 2$, overlapping of the double-sided bands is obtained at every harmonic occurs.

In order to characterize this RF bandwidth capacity in an OFM link, a laser source frequency $\left(\lambda_{0}=1316 \mathrm{~nm}\right)$ was swept by an optical phase modulator with a sweep frequency $f_{\mathrm{sw}}=3 \mathrm{GHz}$, and launched into an MZI with 10-GHz FSR. Different RF subcarriers $f_{\mathrm{sc}}$ ranging from 100 to $1400 \mathrm{MHz}\left(f_{\mathrm{sc}}<f_{\mathrm{sw}} / 2\right.$, in order to avoid overlapping) with RF input power $-4 \mathrm{dBm}$ were employed to modulate the intensity of the light source by a chirpfree Mach-Zehnder IM. After the photodetector, the generated $f_{\mathrm{RF}}=n f_{\mathrm{sw}} \pm f_{\mathrm{sc}}$ subcarriers were measured for the second ( $n=2,6 \mathrm{GHz})$ and the sixth $(n=6,18 \mathrm{GHz})$ harmonics.

As can be seen in Fig. 2(b) and (c), the OFM principle has a flat response for the different RF subcarriers $f_{\mathrm{RF}}$ recovered along with the corresponding harmonic. When $f_{\mathrm{sc}}=f_{\mathrm{sw}} / 2=1500$ $\mathrm{MHz}$, overlapping of the $f_{\mathrm{sc}}$ carried by the adjacent harmonics was occurred. When $f_{\mathrm{sc}}>f_{\mathrm{sw}} / 2$, the recovered $f_{\mathrm{RF}}$ were obtained closer to the adjacent harmonics, and, since overlapping of the harmonic double-side bands occurred, instabilities were observed.

Thus, any low RF subcarrier $f_{\mathrm{sc}}<f_{\mathrm{sw}} / 2$ can be introduced by the IM at the CS, transparently transmitted to the AS, and recovered up-converted along with the desired harmonic. On the condition that this maximum RF bandwidth is not exceeded, different wireless signals can be transmitted simultaneously in a subcarrier multiplexing (SCM) scheme. The up-converted signals obtained after the photodetector can be selected at the same or at different harmonics. This opens the possibility of increasing the cell capacity of a wireless system without the necessity of 


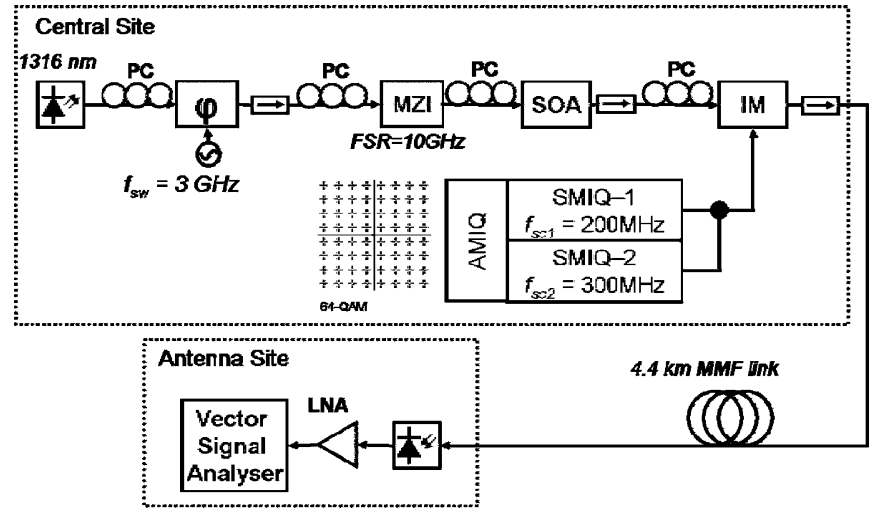

Fig. 3. Experimental setup for multistandard support demonstration (AMIQ: I/Q modulation generator; SMIQ: vector signal generator).

deploying new transceivers at the AS, when the up-converted signals are selected in the same harmonic (only new narrowband RF bandpass filters are necessary after the photodetector to select the new added radio signals, or alternatively, a broadband RF bandpass filter with bandwidth equal to the RF harmonic band can be employed to let pass simultaneously the multiple radio signals). Also, a proper election of the $f_{\mathrm{sw}}$ and $f_{\mathrm{sc}} \mathrm{s}$ at the CS enables the recovery of the different wireless signals at different high RF bands (along with different harmonics) at the AS. In this way, different wireless standards can be simultaneously and transparently transmitted to the same AS by a single OFM link.

\section{MUltistandARD Wireless ACCESS SUPPORT}

In order to demonstrate the feasibility of the multistandard wireless access support by one single OFM link, the experimental setup depicted in Fig. 3 was built.

A laser source frequency $\left(\lambda_{0}=1316 \mathrm{~nm}\right)$ was swept by an optical phase modulator with a sweep frequency $f_{\mathrm{sw}}=3 \mathrm{GHz}$, and launched into an MZI with $10-\mathrm{GHz}$ FSR. The output of the MZI was amplified by a semiconductor optical amplifier (SOA) and launched into a chirp-free Mach-Zehnder IM. The IM was used to introduce two different 64QAM 24-Mb/s signals on low-RF subcarriers $f_{\mathrm{sc} 1}=200 \mathrm{MHz}$ and $f_{\mathrm{sc} 2}=300 \mathrm{MHz}$ (with RF average output power 13 and $16 \mathrm{dBm}$, respectively) into the OFM link. The intensity modulated swept light signal was launched into a 4.4-km MMF, and recovered by a $25-\mathrm{GHz}$ IR photodetector. The output of the photodetector was amplified by a low noise amplifier (LNA) and analyzed by a vector signal analyzer (Rhode \& Schwarz FSQ-40).

At the output of the photodetector, the 64QAM signals at $f_{\mathrm{sc} 1}$ and $f_{\mathrm{sc} 2}$ introduced by the IM at the CS were obtained double-sided along with all the generated harmonics of $f_{\mathrm{sw}}$ at the high frequencies $f_{\mathrm{RF}}=n f_{\mathrm{sw}} \pm f_{\mathrm{sc}}$, at the AS. Fig. 4 shows the spectrum of the second and sixth harmonics carrying simultaneously the up-converted $f_{\mathrm{sc} 1}=200 \mathrm{MHz}$ and $f_{\mathrm{sc} 2}=300$ $\mathrm{MHz}$, double-sided at the high frequencies $f_{\mathrm{RF}}$, which are indicated in Table I. The selection of the up-converted $f_{\mathrm{sc} 1}$ and $f_{\mathrm{sc} 2}$ at different harmonics enables the simultaneous transmission of different wireless standards in the same OFM link. For example,

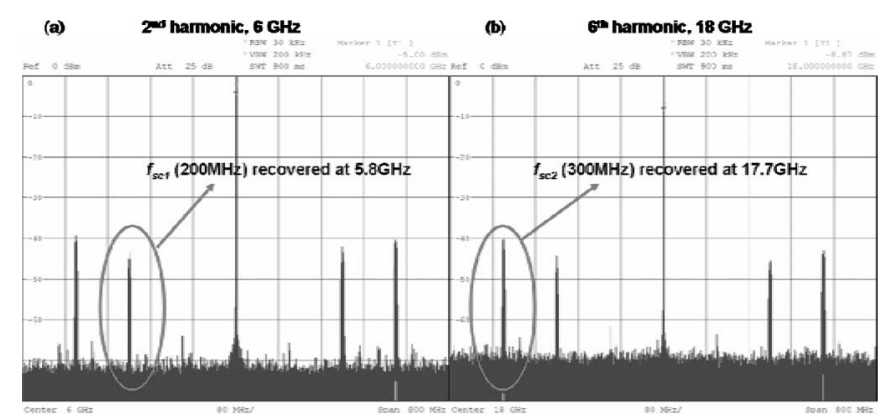

Fig. 4. Simultaneous recovery of input 64QAM signals (200 and $300 \mathrm{MHz}$ ) at (a) $5.8 \mathrm{GHz}$ and (b) $17.7 \mathrm{GHz}$ after $4.4 \mathrm{~km}$ of MMF transmission.

TABLE I

High FREQUENCIES $\left(f_{\mathrm{RF}}\right)$ OBTAINED AT THE SECOND AND SiXTH HARMONICS

\begin{tabular}{c||c|c|c|c}
\hline \multirow{2}{*}{ Input signal } & \multicolumn{2}{c|}{$2^{\text {nd }}$ harmonic $(6 \mathrm{GHz})$} & \multicolumn{2}{c}{$6^{\text {th }}$ harmonic $(18 \mathrm{GHz})$} \\
\cline { 2 - 5 } & Lower band & Upper band & Lower band & Upper band \\
\hline \hline$f_{s c l}=200 \mathrm{MHz}$ & $\mathbf{5 . 8 G H z}$ & $6.2 \mathrm{GHz}$ & $17.8 \mathrm{GHz}$ & $18.2 \mathrm{GHz}$ \\
\hline$f_{s c 2}=300 \mathrm{MHz}$ & $5.7 \mathrm{GHz}$ & $6.3 \mathrm{GHz}$ & $\mathbf{1 7 . 7 G H z}$ & $18.3 \mathrm{GHz}$ \\
\hline
\end{tabular}

the signal introduced by $f_{\mathrm{sc} 1}$ at $f_{\mathrm{RF} 1}=5.8 \mathrm{GHz}$ and the one introduced by $f_{\mathrm{sc} 2}$ at $f_{\mathrm{RF} 2}=17.7 \mathrm{GHz}$ can be selected in order to deliver simultaneously broadband wireless signals in the sub-11 GHz band (e.g., for some wireless LAN systems, like IEEE802.11a) and for fixed broadband wireless access in the $17-\mathrm{GHz}$ band (this is an unlicensed frequency band allocated for fixed broadband wireless access in the national frequency plan of some European countries [12]).

Fig. 5 shows the IQ constellation, I-eye and Q-eye diagrams obtained for the two 64 QAM signals recovered simultaneously at $f_{\mathrm{RF} 1}=5.8 \mathrm{GHz}\left(f_{\mathrm{sc} 1}\right.$, second harmonic $)$ and $f_{\mathrm{RF} 2}=17.7$ $\mathrm{GHz}\left(f_{\mathrm{sc} 2}\right.$, sixth harmonic) after $4.4 \mathrm{~km}$ of MMF transmission. Error vector magnitude (EVM) values of $5.417 \%$ and $5.357 \%$ were measured, respectively, which correspond to SNR values of 25.32 and $25.42 \mathrm{~dB}$, respectively. (These values lie within the boundaries allowed by IEEE 802.11a for the transmitter constellation error of 64QAM signals at the 5-GHz band).

In order to assess the signal degradation occurred due to SCM for the simultaneous transmission of two 64QAM signals, a single 64QAM signal at $f_{\mathrm{sc} 2}=300 \mathrm{MHz}$ was introduced into the OFM link and transmitted over the $4.4 \mathrm{~km}$ of MMF under the same experimental conditions. The upconverted 64QAM signals recovered at $5.7 \mathrm{GHz}$ (second harmonic) and $17.7 \mathrm{GHz}$ (sixth harmonic) experienced an EVM of $3.185 \%(\mathrm{SNR}=29.94 \mathrm{~dB})$ and $3.199 \%(\mathrm{SNR}=29.90 \mathrm{~dB})$, respectively.

Thus, a signal degradation of around $4.5 \mathrm{~dB}$ occurs due to simultaneous transmission of two 64QAM signals (SCM) in the same OFM link with $4.4 \mathrm{~km}$ of MMF. This degradation is caused first by the nonlinear response of the IM for different subcarrier frequencies and the effective IM index per channel, which varies with the number of channels (number of 64QAM signals) applied; second, intermodulation products in the IM and undesired beating interference between the subcarriers occurs in the optical up-conversion and transmission of the radio signals, which contributes to the degradation of the 64QAM signals. 


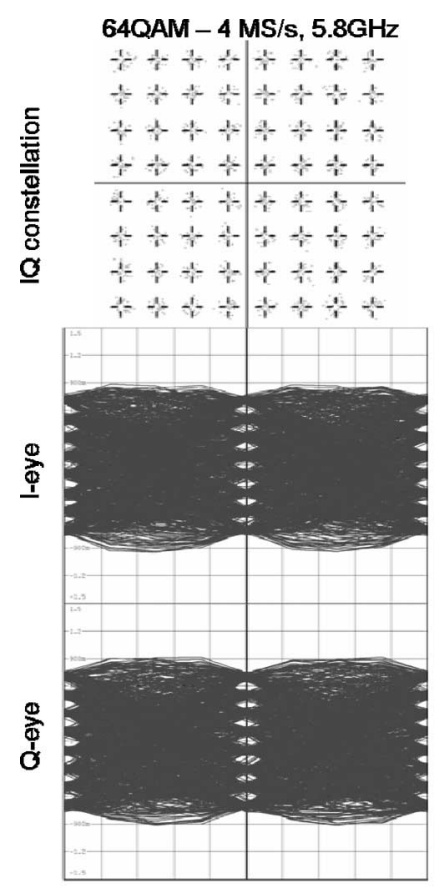

(a)

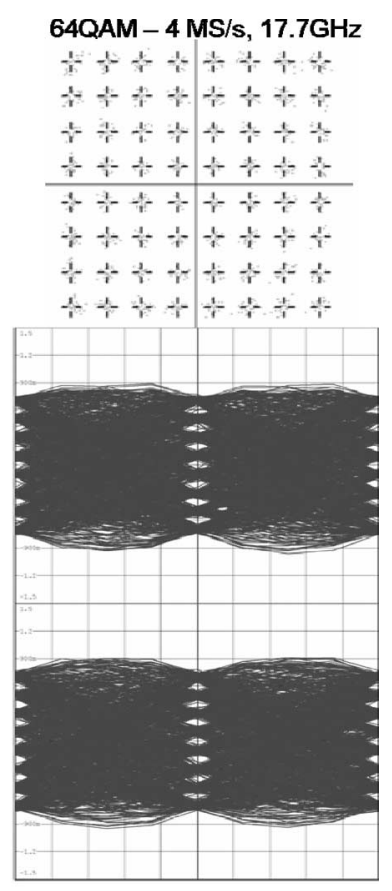

(b)
Fig. 5. IQ constellation, I-eye, and Q-eye diagrams obtained for the two 64QAM signals obtained simultaneously at (a) $5.8 \mathrm{GHz}$ and (b) $17.7 \mathrm{GHz}$ after $4.4 \mathrm{~km}$ of MMF transmission.

\section{DYNAMIC RADIO LINK ADAPTATION SUPPORT}

In wired data transmission systems, the response of the physical medium can be well predicted and estimated. On the contrary, wireless data transmission systems are characterized by a time-varying environment due to several factors (changing surroundings, variation of the atmosphere conditions, fading, noise level, interferences, and link distance). Besides, time-varying application requirements (such as data rate, quality of service (QoS), number of users and transmit power) determine the transmission efficiency of these kinds of systems. Therefore, dynamic radio link adaptation to the physical radio medium is a key feature in wireless systems. An RoF distribution system has to guarantee this adaptability without incurring in additional signal degradation along the optical path caused by the radio link adaptation procedures. This means that the response of the optical RoF link has to be transparent and as independent as possible to the radio link adaptation procedures.

Adaptation of the transmission to the radio conditions can be achieved by different methods carried out at different places of the protocol stack [13]. These methods are based mainly on the variation of one or more transmission features according to the radio conditions, in order to improve and/or optimize the system performance and QoS in terms of radio resource utilization, mean data throughput, and spectrum efficiency among others. Some of these transmission features are indicated in Table II, classified in three main groups.

Usually, the overall adaptation framework is a rather complex set of mechanisms that try to satisfy multiple conflicting objectives and to optimize diverse cost functions. It defines and identifies relationships between application requirements and radio
TABLE II

TRANSMISSION FEATURES FOR DYNAMIC RADIO LINK ADAPTATION IN AN ROF DISTRIBUTION SYSTEM

\begin{tabular}{l||l||c|c}
\hline Level & Adaptation & CS & AS \\
\hline \hline Link/MAC & $\begin{array}{l}\text { Frame length } \\
\text { Channel coding }\end{array}$ & $\sqrt{ }$ & $\times$ \\
\hline Baseband & $\begin{array}{l}\text { Symbol coding } \\
\text { Modulation } \\
\text { Spreading gain } \\
\text { Equalization }\end{array}$ & $\sqrt{ }$ & $\times$ \\
\hline RF & $\begin{array}{l}\text { Transmit power } \\
\text { Carrier frequency }\end{array}$ & $\sqrt{ }$ & $\sqrt{ }$ \\
\hline
\end{tabular}

parameters. It looks for the most appropriate place to perform the adaptation, evaluating how these transmission features affect the cost functions, coordinating, and controlling the adaptation of the various transmission features. In general, given certain radio characteristics (e.g., power), capabilities (e.g., modulation and coding scheme), time-varying channel constraints, and particular QoS requirements, a wireless system tries to optimize measures like bit error rate (BER), data throughput, delay and spectrum efficiency by means of selecting and adapting the different link and physical layer parameters involved in the transmission.

For example, link adaptation to maximize mean data throughput can be achieved by means of dynamically varying the modulation scheme accordingly to the carrier-to-interferer ratio (CIR), BER, and packet error rate (PER) variations, i.e., using M-ary modulation schemes with high CIR and low BER/PER values (good radio conditions), while employing binary modulation schemes and more robust channel coding with low CIR and high BER/PER values (bad radio conditions).

In an RoF link, it becomes important to identify where the different levels of adaptation take place and from where they can be controlled. Consolidating the signal processing load at the CS, while maintaining the radio frequency modules at the AS, gives a clear differentiation for the adaptation level separation (Table II): Link/MAC and baseband adaptation can be controlled from the CS (where it takes place), whereas the RF adaptation may occur either at the CS or at the AS. In this last case, adaptive remote AS configuration might be necessary. Hence, a tradeoff between antenna-station-simplicity and minimum level of antenna-station-intelligence need arises, when deciding the most appropriate location to perform and control the RF adaptation.

\section{A. Link Adaptation at the CS: Dynamic Frequency Selection}

Dynamic frequency selection is performed by some wireless systems in order to make an equal usage of available frequencies under the consideration of avoiding the interference of other devices using the same spectrum. The interference may arise from adjacent cells using the same frequency or from other systems working in the same frequency band. Other wireless systems may use frequency hopping techniques in order to overcome fading effects. Thus, the RoF distribution system design has to consider an appropriate carrier frequency adaptation support.

By employing OFM, this carrier frequency adaptation is achieved straightforwardly from the CS. At the CS, the radio 


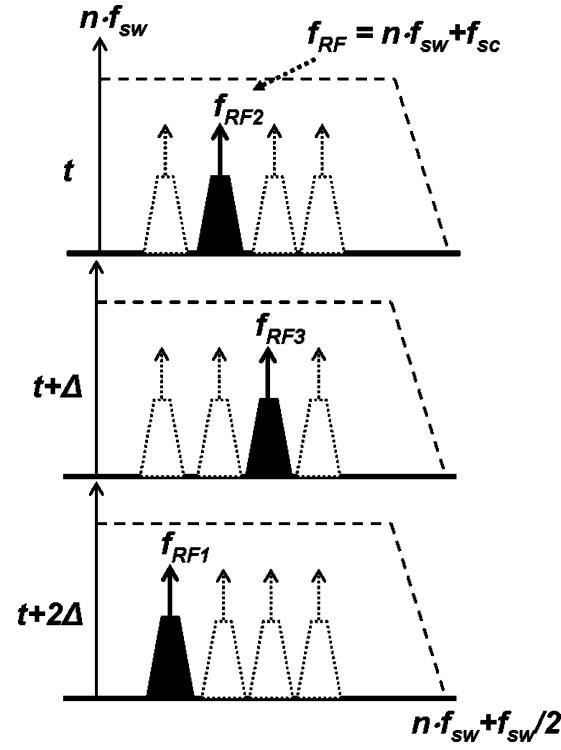

Fig. 6. Link adaptation at the CS. Dynamic frequency allocation.

data signal is put onto a low-frequency subcarrier $f_{\mathrm{sc}}$. This subcarrier is then used to modulate the intensity of the swept light source as shown in Section II. At the AS, the radio data signal is recovered at the desired $f_{\mathrm{RF}}$ along with the corresponding harmonic. Thus, adjusting the low-frequency subcarrier $f_{\mathrm{sc}}$ at the $\mathrm{CS}\left(f_{\mathrm{sc} 2}\right.$ at $t, f_{\mathrm{sc} 3}$ at $t+\Delta, f_{\mathrm{sc} 1}$ at $\left.t+2 \Delta \ldots\right)$ will result on the same $f_{\mathrm{RF}}$ carrier variations $\left(f_{\mathrm{RF} 2}\right.$ at $t, f_{\mathrm{RF} 3}$ at $t+\Delta, f_{\mathrm{RF} 1}$ at $t+2 \Delta \ldots)$ at the AS, without increasing the AS complexity. This is depicted in Fig. 6. The maximum frequency deviations that can be achieved with this scheme are limited by the maximum RF bandwidth $\left(f_{\mathrm{sw}} / 2\right)$ allowed by OFM and a broadband bandpass filter letting through those deviations.

\section{B. Link Adaptation at the AS: Transmit Power Control}

Power control algorithms are a common mechanism of RF adaptation for diverse objectives. They basically consist in varying the transmit power at the antenna connector according to the radio link conditions, in order to reduce the co-channel and adjacent channel interference. In consequence, they allow improving the link quality, the radio resource utilization, the frequency reuse, and the radio coverage.

In an RoF link, the transmit power could be adjusted either at the CS or at the AS. Targeting the AS simplicity, the transmit power can be regulated at the CS according to the power control procedure. Provided that the optical link have a linear response, the variations of transmitted power at the CS would result in variations of the same range and magnitude at the AS by compensating the losses incurred along the optical path with a proper (and fixed gain) amplification. Despite the simplicity of this method, it may also yield some other inconveniences as follows.

Power control procedures are usually executed in tight relationship with other baseband adaptation methods. For example, given a transmit power level, the modulation and coding scheme can be dynamically adjusted to accomplish with a pre- defined PER requirement. Under good radio conditions, the PER requirement can be easily fulfilled by employing coding schemes with little redundancy for error correction, whereas when the radio conditions worsen, the channel coding has to be downgraded to more robust schemes that add more protection (and consequently, providing lower nominal data rate), maintaining the same power level. If, under these conditions, certain criteria are satisfied, the overall adaptation framework may decide to increase the transmit power level, so that the modulation and coding scheme can be upgraded again to achieve a higher data rate. Also the other way around, the overall adaptation framework may decide to maintain the channel coding scheme while varying the transmit power levels, and perform a modulation and coding scheme upgrade (downgrade) only once the minimum (maximum) transmit power level has been reached.

In an RoF link where the transmit power adaptation is performed at the CS, varying the transmit power may entail that the transmission falls outside the dynamic range of the link. This would yield a modulation and coding scheme downgrade triggered by the optical link quality, disregarding the radio conditions and even if the radio conditions have not changed. Consequently, the system performance decreases in terms of data throughput.

To avoid this, transmit power adaptation can be implemented remotely at the AS by dynamic adaptive amplification, after having transmitted the signals over the optical link with optimal power conditions. In this approach, the optical link becomes independent of radio power control variations, and as a result, the requirement of optical link transparency is better fulfilled. This purpose, however, results in an increased complexity of both the AS and the distribution system design itself. On the one hand, controlled gain amplification is needed at the AS before radiation. On the other hand, optical resources must be granted to transmit the power controlling signals from the CS to the remote adaptive amplifier simultaneously with the radio signals. This implies the introduction of an additional signaling control channel together with the data channels for remote dynamic power control at the AS, alleviating the requirement of high dynamic range.

In a more general approach, the introduction of a control channel in the same optical link may enable other mechanisms for remote antenna configuration and controlling during network optimization and dynamic resource allocation.

\section{Simultaneous Transmission of Data and Control Channels}

As a proof of concept, an experiment similar to the one depicted in Fig. 3 was set up, in order to demonstrate the feasibility of inserting an in-band control channel together with the radio data channel employing the OFM principle (Fig. 7).

The data channel consisted in a 64QAM 24-Mb/s signal onto a low RF subcarrier $f_{\mathrm{sc}}=400 \mathrm{MHz}$ at the CS. The control channel consisted in a baseband NRZ bit pattern (PBRS $2^{23}-1$ ) at 50, 100, and $150 \mathrm{MHz}$. The wideband output of the pattern generator was truncated by a $120-\mathrm{MHz}$ low-pass filter before transmission, in order to avoid dramatic interference 


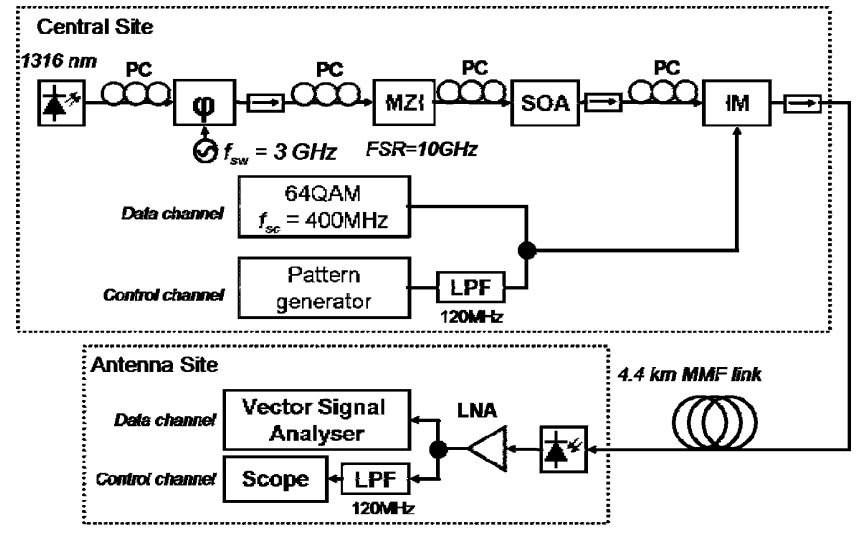

Fig. 7. Experimental setup for simultaneous transmission of a control channel and a radio data channel.
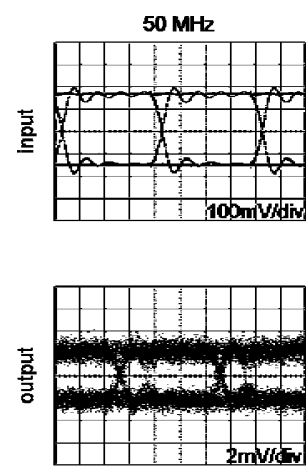

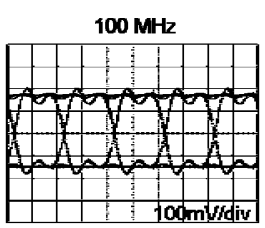

(a)

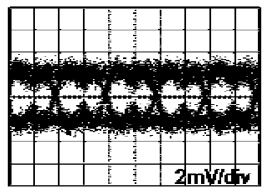

(b)
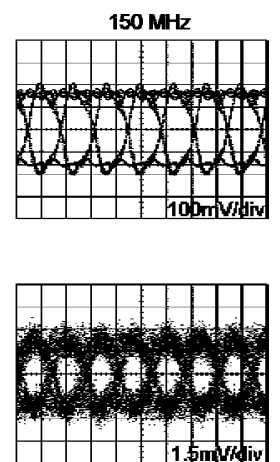
the AS.

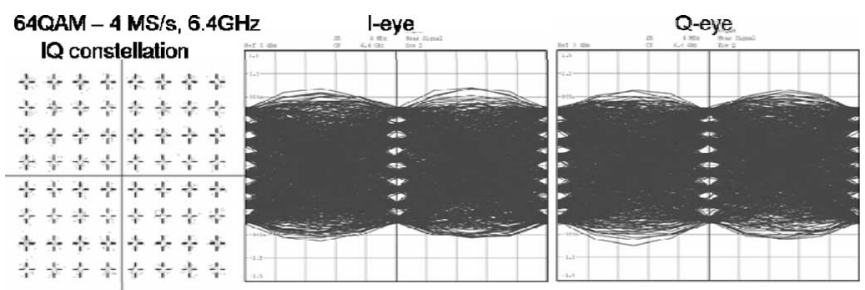

Fig. 9. 64QAM data channel recovered at $6.4 \mathrm{GHz}$ (second harmonic) after $4.4 \mathrm{~km}$ of MMF

between harmonics and with the simultaneously transmitted 64QAM signal in the OFM link. The composite signal was employed to modulate the intensity of the swept light source by a chirp-free Mach-Zehnder IM. The resulting optical signal was launched into a 4.4-km MMF link and recovered by a 25-GHz IR photodetector.

At the AS, the control channel was visualized by a scope after having filtered the photodetector output by a $120-\mathrm{MHz}$ low-pass filter. Fig. 8 shows the eye diagrams obtained for the 50, 100, and $150-\mathrm{MHz}$ control channel recovered after $4.4 \mathrm{~km}$ of MMF at the AS.

The simultaneously transmitted data channel was visualized by a vector signal analyzer at the AS. The 64QAM signal introduced at the CS at $f_{\mathrm{sc}}=400 \mathrm{MHz}$ was recovered after the photodetector at $f_{\mathrm{RF}}=6.4 \mathrm{GHz}$ (second harmonic). Fig. 9 shows the IQ constellation, I-eye and Q-eye diagrams obtained. An EVM value of $5.447 \%$, corresponding to a SNR of 25.28 $\mathrm{dB}$, was observed. This means a signal degradation of $\sim 4.6$ $\mathrm{dB}$ with respect to the single transmission of the data channel $(\mathrm{SNR}=29.9 \mathrm{~dB}$, see Section III). The variation of the control channel bit rate $(50-150 \mathrm{MHz})$ did not cause any changes in the data channel recovery.

\section{CONCLUSION}

RoF distribution antenna systems are foreseen as a flexible, bandwidth-efficient, and cost-effective option for fiber-based wireless access infrastructure, especially in in-building and business environments. In order to provide a reliable $\mathrm{RoF}$ infrastructure for future broadband wireless access, multistandard transmission and dynamic radio link adaptation strategies have to be supported. OFM is a simple and cost-effective method for this purpose.

By employing OFM, the simultaneous delivery at the AS of 64QAM signals at $5.8 \mathrm{GHz}$ (for wireless access in the sub$11 \mathrm{GHz}$ band) and at $17.7 \mathrm{GHz}$ (for fixed broadband wireless access in the $17-\mathrm{GHz}$ band) after 4.4-km of MMF is demonstrated experimentally. This is achieved by remotely introducing the two 64QAM signals at low-frequency subcarriers (200 and $300 \mathrm{MHz}$ ) from the CS. EVM values below 5.6\% (as recommended by the IEEE802.11a standard for 64QAM signals in the $5-\mathrm{GHz}$ band) were measure for both signals.

OFM also enables a flexible mechanism for dynamic radio link adaptation support. Carrier frequency adaptation can be easily performed from the CS by tuning low-frequency subcarriers. Transmit power can be remotely controlled from the CS and adjusted at the AS. For this purpose, an in-band control channel has to be transmitted simultaneously with the wireless data channels from the CS to the AS. The experiments show the successful simultaneous delivery of a 64QAM signal at $6.4 \mathrm{GHz}$ and a baseband NRZ bit pattern (PBRS $2^{23}-1$ ) at 50, 100, and $150 \mathrm{MHz}$ after $4.4 \mathrm{~km}$ of MMF by a single OFM link.

These experimental results have prospects for future broadband wireless access infrastructure employing RoF links, enabling the coexistence of different wireless standards at the same AS and a flexible mechanism for RF link adaptation.

\section{REFERENCES}

[1] W. Fischer, "Fiber to the home deployments in Europe-architectural and technological considerations," presented at the 10th Eur. Conf. Netw. Opt. Commun., London, U.K., 2005.

[2] C. Howson, K. Rimstad, and M. Topman, "Next generation wireless access solutions," presented at the 10th European Conf. Netw. Opt. Commun., London, U.K., 2005.

[3] D. Wake, "Trends and prospects for radio over fibre picocells," in $M W P$ ' 02 , 2002 IEEE Int. Top. Meeting Microw. Photon., pp. 21-24.

[4] J. E. Mitchell, "Performance of OFDM at $5.8 \mathrm{GHz}$ using radio over fibre link," Electron. Lett., vol. 40, no. 21, pp. 1353-1354, Oct. 2004.

[5] C. Lim, A. Nirmalathas, M. Attygalle, D. Novak, and R. Waterhouse, "On the merging of millimeter-wave fiber-radio backbone with 25-GHz WDM ring networks," J. Lightw. Technol., vol. 21, no. 10, pp. 2203-2210, Oct. 2003.

[6] Y. K. Seo, J. H. Seo, and W. Y. Choi, "Photonic frequency-upconversion efficiencies in semiconductor optical amplifiers," IEEE Photon. Technol. Lett., vol. 15, no. 5, pp. 751-753, May 2003. 
[7] L. A. Johansson and A. J. Seeds, "Generation and transmission of millimeter-wave data-modulated optical signals using an optical injection phase-lock loop," J. Lightw. Technol., vol. 21, no. 2, pp. 511-520, Feb. 2003.

[8] T. Koonen, A. Ng'oma, P. Smulders, H. van den Boom, I. T. Monroy, and D. G. Khoe, "In-house networks using multimode polymer optical fiber for broadband wireless services," Photon. Netw. Commun., vol. 5, no. 2, pp. 177-187, 2003.

[9] T. Koonen, A. Ng'oma, M. G. Larrode, F. Huijskens, I. T. Monroy, and D. G. Khoe, "Novel cost-efficient techniques for microwave signal delivery in fibre-wireless networks," presented at the 30th Eur. Conf. Opt. Commun., Stockholm, Sweden, 2004.

[10] M. G. Larrodé, A. M. J. Koonen, J. J. Vegas Olmos, G. J. Rijckenberg, L. D. Bao, and I. Niemegeers, "Transparent transport of wireless communication signals in radio-over-fibre systems," presented at the 10th Eur. Conf. Netw. Opt. Commun., London, U.K., 2005.

[11] N. G. Walker, D. Wake, and I. C. Smith, "Efficient millimetre-wave signal generation through FM-IM conversion in dispersive optical fiber links," Electron. Lett., vol. 28, no. 21, pp. 2027-2028, Oct. 1992.

[12] Ministerie van Economische Zaken (The Netherlands), Directoraat Generaal Telecommunicatie en Post, Agentschap Telecom, "Nationaal Frequentie Plan 2005 (NFP)," Jan. 2005.

[13] M. Srivastava, (2002) Lecture on Wireless Link Adaptation, Univ. California at Los Angeles (UCLA)., [Online]. Available: http://nesl. ee.ucla.edu/courses/ ee206a/2002s/lectures/L07.ppt

M. García Larrodé (S’04) was born in Zaragoza, Spain, in 1977. She received the M.Sc. degree in telecommunications engineering from the Centro Politécnico Superior, University of Zaragoza, Zaragoza, Spain, in 2001. Currently, she is working toward the Ph.D. degree in the area of broadband wireless access networks employing radio over fiber techniques at the COBRA Research Institute, Eindhoven University of Technology, Eindhoven, The Netherlands.

From 2000 to 2004, she was with Siemens AG as a Systems Engineer in mobile radio access networks, focusing on radio resource management, signaling, and performance evaluation of GSM/GPRS/EDGE and UMTS networks.
A. M. J. Koonen (M'00-SM'01) was born in Oss, The Netherlands, on October 20, 1954. He received the M.Sc. degree (cum laude) in electrical engineering from Eindhoven University of Technology, Eindhoven, The Netherlands, in 1979.

In 1979, he joined Philips' Telecommunicatie Industrie, part of which has since 1984 become Lucent Technologies Network Systems, The Netherlands. He worked on high-speed transmission systems and optical fiber systems for hybrid access networks. From 1987 to 2000, he was a Technical Manager at the Forward Looking Work Department, Bell Laboratories, Lucent Technologies Network Systems. From 1991 to September 2000, he was a part-time Professor at the University of Twente, holding a Chair on Photonic Networks. In September 2000, he became a part-time Professor at the Technical University of Eindhoven, and since January 2001, he has been a full-time Professor, holding a Chair on Broadband Communication Networks in the Department Telecommunication Technology and Electromagnetics. In 1999, he was appointed as the Bell Labs Fellow.

J. J. Vegas Olmos (S'04) was born in Barcelona, Spain, in 1978. He received the B.Sc. degree in telecommunications engineering and the M.Sc. degree in electronics engineering from the Universitat Politecnica de Catalunya, Barcelona, Spain, in 2001 and 2003, respectively. He also received the Licenciature in Business Administration from the Universitat Oberta de Catalunya in 2005 Currently, he is working toward the Ph.D. degree in label-controlled optical routing nodes in the Electro-Optical Communications Group, Eindhoven University of Technology, Eindhoven, The Netherlands. 